\title{
Integral Reinforcement Learning with Explorations for Continuous-Time Nonlinear Systems
}

\author{
Jae Young Lee \\ School of Electrical and \\ Electronics Engineering \\ Yonsei University, \\ Seoul 120-749, Korea \\ Email: jyounglee@yonsei.ac.kr
}

\author{
Jin Bae Park \\ School of Electrical and \\ Electronics Engineering \\ Yonsei University \\ Seoul 120-749, Korea \\ Email: jbpark@yonsei.ac.kr
}

\author{
Yoon Ho Choi \\ Department of Electronic \\ Engineering \\ Kyonggi University \\ Kyonggi-Do 443-760, Korea \\ Email: yhchoi@kyonggi.ac.kr
}

\begin{abstract}
This paper focuses on the integral reinforcement learning (I-RL) for input-affine continuous-time (CT) nonlinear systems where a known time-varying signal called an exploration is injected through the control input. First, we propose a modified I-RL method which effectively eliminates the effects of the explorations on the algorithm. Next, based on the result, an actor-critic I-RL technique is presented for the same nonlinear systems with completely unknown dynamics. Finally, the leastsquares implementation method with the exact parameterizations is presented for each proposed one which can be solved under the given persistently exciting (PE) conditions. A simulation example is given to verify the effectiveness of the proposed methods.
\end{abstract}

\section{INTRODUCTION}

Reinforcement learning (RL) is a class of computational method to interactively find an optimal policy based on the decision/control input and the output observed data associated with the unknown environments [1], [2]. At the beginning, the researches on RL were focused only on the Markov decision process (MDP) environment with the discrete states and decisions. In those methods, the infinitely many explorationsthe injection of a random policy (e.g. $\epsilon$-greedy) for some time instant-are essential for the convergence to the optimal policy, known as exploitation and exploration dilemma [1].

As those RL methods for MDP have been studied and matured, the RL techniques for dynamical systems also have had much attention by the researchers in the fields of computational intelligence and control theory [2]-[19]. This kind of RL methods are developed at first for discrete-time (DT) dynamical systems [2]-[9], and some of the methods are proven to be stable and converge to the optimal solution [4], [6]-[9]. In these researches, some exploration-like concepts such as state resetting [7], covariance resetting, and injection of a small probing noise signal through the control input [6], [8] are used for maintaining the persistently exciting (PE) condition for the convergence and computability of the parameters. Especially, in the model-free RL scheme [6], [8], it is shown that the small probing noise is essentially required to maintain the given excitation or linear independence conditions.

In continuous-time (CT) framework, the RL algorithms were developed without any proof of stability and convergence at first [10], [11], and after the pioneering work give in
[12]-[14], a class of RL methods, named as integral RL (IRL), was developed for nonlinear input-affine CT systems [4], [15]. Those I-RL methods are based on the integral temporal difference (I-TD) and do not require the knowledge of the system's drift dynamics during the iteration. Here, the iteration is related with finding the online solution of the associated Hamilton-Jacobi-Bellman (HJB) equation to solve the nonlinear optimal control problems in online fashion [4], [15]. Nonlinear optimal control problems are generally hard to solve even in offline fashion due to the difficulty arising from the HJB equation. Moreover, for the I-RL derived from policy iteration [15], the stability and convergence to the optimal solution has been proven under initial stabilizing policy.

However, all those I-RL methods require the input coupling dynamics to update the next policy and there is no way to excite the state variables during the online iteration. For the other recently-developed RL methods [16], [17], the latter is solved by using the probing noise signal. On the contrary, at least the same assumption of the known input coupling dynamics is needed for the update of the policy by adaptation.

In this paper, based on the I-RL given in [15], two I-RL algorithms are proposed for CT nonlinear systems where a known time-varying signal called an exploration is injected through the control input. Specifically,

- the modified I-RL is presented by incorporating the explorations into the algorithm; the method effectively eliminate the effects of the explorations;

- based on the modified one, the second one called "modelfree I-RL" technique is presented for the same nonlinear systems with completely unknown dynamics.

The result shows that in both algorithms, the input-coupling dynamics is directly coupled with the exploration injected by the control input. Furthermore, for the proposed methods, we present the least-squares implementation technique with the exact parameterizations, which can be solved under the suitable PE condition. A simulation example is given to verify the effectiveness of the proposed methods. 


\section{Nonlinear Optimal Control Problems}

In this paper, we consider the following CT input-affine nonlinear system:

$$
\dot{x}=f(x)+g(x) u .
$$

with the following performance index:

$$
V(x(t), u(\cdot))=\int_{t}^{\infty} r(x(\tau), u(\tau)) d \tau
$$

where $x \in \mathbb{R}^{n}$ and $u \in \mathbb{R}^{m}$ are the state variable and control input; $f(x) \in \mathbb{R}^{n}$ and $g(x) \in \mathbb{R}^{n \times m}$ are nonlinear functions; $r(x, u):=Q(x)+u^{T} R u>0$ with positive definite function $Q(x)$ and positive definite matrix $R$. Here, we assume that $f(0)=0$ and $f(x)+g(x) u$ is locally Lipschitz on a set $\Omega \subseteq \mathbb{R}^{n}$ containing the origin, or equivalently, there is a policy $u=\mu(x) \in \mathbb{R}^{m}$ making (1) asymptotically stable. For the performance criteria (2), the admissible policy is defined as follows:

Definition 1: A control policy $u=\mu(x)$ with $\mu(0)=0$ is said to be admissible with respect to (2) on $\Omega$ if $\mu(x)$ is continuous on $\Omega$, stabilizes the system (1), and yields a finite value function $V\left(x_{t}\right)=\left.V\left(x_{t}, u(\cdot)\right)\right|_{u=\mu(x)}$.

For the system (1) and the value function (2), let $\mathcal{U}(\Omega)$ be the set of all admissible policy on $\Omega$. Then, for an admissible policy $\mu(x) \in \mathcal{U}(\Omega)$, if the associated value function $V_{\mu}(x):=$ $\left.V(x, u(\cdot))\right|_{u=\mu}$ is continuously differentiable, then, we obtain the following Lyapunov equation for the nonlinear system (1):

$$
r(x, \mu(x))+\left(\frac{\partial V_{\mu}}{\partial x}\right)^{T}(f(x)+g(x) \mu(x))=0,
$$

which is actually the infinitesimal version of (2) and implies $\dot{V}_{\mu}(x)=-r(x, \mu(x))$ along the trajectory of (1). Note that by $\dot{V}_{\mu}(x) \leq 0$ and $V_{\mu}(x)>0, V_{\mu}(x)$ is obviously a Lyapunov function for the system (1). Here, the admissible policy $u=$ $\mu(x) \in \mathcal{U}(\Omega)$ guarantees $V_{\mu}(x)$ is continuous differentiable by the continuity of $\mu(x)$ on $\Omega$ (see the equation (2) with the definition of $r(x, u))$.

The optimal policy $\mu^{*}(x)$ is an admissible policy which minimizes the performance index (2). By minimizing the Hamiltonian $H\left(x, \mu, V^{*}\right)$ defined by

$$
H\left(x, \mu, V^{*}\right):=r(x, \mu)+\left(\frac{\partial V^{*}}{\partial x}\right)^{T}(f(x)+g(x) \mu(x))
$$

among all admissible policies $\mu \in \mathcal{U}(\Omega)$, the optimal policy $u^{*}=\mu^{*}(x)$ can be obtained as

$$
u^{*}=-\frac{1}{2} R^{-1} g^{T}(x)\left(\frac{\partial V^{*}}{\partial x}\right) .
$$

Substituting this into (3) yields the well-known HamiltonJacobi-Bellman (HJB) equation:

$$
\begin{aligned}
& Q(x)+\left(\frac{\partial V^{*}}{\partial x}\right)^{T} f(x)-\frac{1}{4}\left(\frac{\partial V^{*}}{\partial x}\right)^{T} g(x) R^{-1} g^{T}(x) \frac{\partial V^{*}}{\partial x}=0, \\
& V^{*}(0)=0 .
\end{aligned}
$$

Here, the existence of $V^{*}$ satisfying (5) is the necessary and sufficient condition for the optimal control problem. Normally it is generally hard and in some cases, impossible to obtain the solution $V^{*}$ of the HJB. Instead of directly solving HJB, one can obtain the solution $V^{*}$ by successively solving the nonlinear Lyapunov equation (3) and update the policy $\mu$ [14], [20]. This successive approximation is known as policy iteration [15] and demonstrated as follows:

\section{Algorithm 1: Policy Iteration (Successive Approximation)}

1: Start with an initial admissible policy $\mu_{0}, i \leftarrow 0$

2: Policy Evaluation: Solve (6) for the positive definite $V_{\mu_{i}}$ :

$$
r\left(x, \mu_{i}(x)\right)+\left(\frac{\partial V_{\mu_{i}}}{\partial x}\right)^{T}\left(f(x)+g(x) \mu_{i}(x)\right)=0,
$$

3: Policy Improvement: Update the next policy $\mu_{i+1}(x)$ by

$$
\mu_{i}(x)=-\frac{1}{2} R^{-1} g^{T}(x)\left(\frac{\partial V_{\mu_{i-1}}}{\partial x}\right)
$$

4: $i \leftarrow i+1$

5: Repeat 2-4 until convergence is met.

Remark 1: In [14] and [20], it was shown that as the iteration goes on by Algorithm 1, $V^{\mu_{i}}$ and $\mu_{i}(x)$ converge to the optimal solution $V^{*}$ and $\mu^{*}(x)$ with the monotonicity property

$$
0<V_{\mu_{i+1}} \leq V_{\mu_{i}} .
$$

\section{INTEGRAL REINFORCEMENT LEARNING BASED ON POLICY ITERATION}

In this section, the nonlinear I-RL given in [15] is briefly discussed and then, propose two variants of the algorithmthe one called the I-RL with explorations (Algorithm 3), and the other named as I-RL with completely unknown dynamics (Algorithm 4). Note that all the I-RL methods described in [4] are based on the following integral temporal difference (I-TD) error for a given admissible policy $\mu \in \mathcal{U}(\Omega)$ :

$$
V_{\mu}\left(x_{t}\right)=\int_{t}^{t+T} r\left(x(\tau), u_{i}(\tau)\right) d \tau+V_{\mu}\left(x_{t+T}\right),
$$

which can be actually obtained by integrating the Lyapunov equation (3) from $t$ to $t+T$. Among the I-RL methods [4], the I-RL related with this paper is the one combined with policy iteration and is shown in the next page [15].

As you can see, this I-RL algorithm is partially model-free, i.e., the system dynamics $f(x)$ is not needed to evaluate both $V_{i}$ and $u_{i}$. Moreover, Vrabie et al. [15] have proven that the evaluated value function $V_{i}(x)$ equals to the value function $V_{\mu_{i}}(x)$ for the $i$-th policy $u_{i}=\mu_{i}(x)$, the equivalence between the I-RL (Algorithm 2) and the policy iteration (Algorithm 1). Therefore, by the properties of Algorithm 1, Algorithm 2 guarantees the stability of the closed-loop system and convergence to the optimal solution under initial stabilizing policy $u_{0}=\mu_{0}(x)$. 


\section{Algorithm 2: I-RL (w/o Explorations)}

1: Start with an initial admissible policy $u_{0}=\mu_{0}(x), i \leftarrow 0$

\section{2: Policy Evaluation:}

For the system (1) with $u=u_{i}$, find $V_{i}(x)$ satisfying

$$
V_{i}\left(x_{t}\right)=\int_{t}^{t+T} r\left(x(\tau), u_{i}(\tau)\right) d \tau+V_{i}\left(x_{t+T}\right)
$$

\section{3: Policy Improvement:}

$$
u_{i}=\mu_{i}(x)=-\frac{1}{2} R^{-1} g^{T}(x)\left(\frac{\partial V_{i-1}}{\partial x}\right)
$$

4: $i \leftarrow i+1$

5: Repeat 2-4 until convergence is met.

In the sequel, two I-RL algorithms are proposed both of which are able to explore the state-space $\Omega \subseteq \mathbb{R}^{n}$. To deal with the explorations, consider the following nonlinear system explored by a known bounded piecewise continuous signal $e_{t}$ :

$$
\dot{x}=f(x)+g(x)\left[u+e_{t}\right] .
$$

We call this known signal $e_{t}$ an exploration $e_{t}$. The following lemma is useful in deriving the I-RL algorithms for the system (11) with an exploration $e_{t}$ :

Lemma 1: Under the admissible policy $u=\mu(x) \in \mathcal{U}(\Omega)$, if the state $x$ is generated by the system (11), then, the value function $V_{\mu}(x)$ satisfies the following integral equation for all bounded piecewise continuous exploration $e_{t}$ :

$$
\begin{aligned}
V_{\mu}\left(x_{t}\right)+\int_{t}^{t+T} & \left(\frac{\partial V_{\mu}}{\partial x}\right)^{T} g(x) e_{\tau} d \tau \\
& =\int_{t}^{t+T} r\left(x_{\tau}, \mu\left(x_{\tau}\right)\right) d \tau+V_{\mu}\left(x_{t+T}\right) .
\end{aligned}
$$

Proof: Assume the policy $u=\mu(x)$ is admissible. Then, the value function $V_{\mu}(x)$ is finite and satisfies the Lyapunov equation (3). By adding $\left(\partial V_{\mu} / \partial x\right)^{T} g(x) e_{t}$ to both sides, (3) can be rewritten as

$$
\begin{aligned}
\left(\frac{\partial V_{\mu}}{\partial x}\right)(f(x) & \left.+g(x)\left[\mu(x)+e_{t}\right]\right) \\
& =-r(x, \mu(x))+\left(\frac{\partial V_{\mu}}{\partial x}\right)^{T} g(x) e_{t} .
\end{aligned}
$$

Then, integrating (13) from $t$ to $t+T$ along the trajectory generated by (11), we obtain the integral equation (12) where the integral is well-defined since $V_{\mu}(x)$ and the interval $[t, t+$ $T$ ] are finite and $e_{t}$ is bounded and piecewise continuous.

Now, the applications of Lemma 1 to the value function $V_{\mu_{i}}(x)$ evaluated by (6) shows that $V_{\mu_{i}}(x)>0$ in Algorithm 1 always satisfies (12) with $\mu=\mu_{i}$. This provides the subequivalence of the policy iteration (Algorithm 1) to the I-RL method (Algorithm 3). That is, if $\left(V_{i}, u_{i}\right)$ are generated by
Algorithm 1 with $V_{i}=V_{\mu_{i}}$ and $u_{i}=\mu_{i}$, then it must satisfy the equations (14) and (15) in Algorithm 3 shown as follows:

\section{Algorithm 3: I-RL with Explorations}

\section{Policy Evaluation:}

$$
\begin{aligned}
V_{i}\left(x_{t}\right)+\int_{t}^{t+T} & \left(\frac{\partial V_{i}}{\partial x}\right)^{T} g(x) e_{\tau} d \tau \\
= & \int_{t}^{t+T} r\left(x(\tau), u_{i}(\tau)\right) d \tau+V_{i}\left(x_{t+T}\right)
\end{aligned}
$$

Policy Improvement:

$$
u_{i}(t)=-\frac{1}{2} R^{-1} g^{T}(x)\left(\frac{\partial V_{i-1}}{\partial x}\right)
$$

This is actually the nonlinear version of the policy iteration given in [18] with an exploration. For simplicity, the detailed codes of Algorithm 3 are omitted which are exactly equal to those in Algorithms 2. Here, the difference due to the non-zero exploration $e_{t}$ lies in the equation (14) of the policy evaluation. Assuming $e_{t} \equiv 0$, one can see that Algorithm 3 turns out to be equal to Algorithm 1 exactly.

Note that in both of the policy evaluation (14) and improvement (15) of Algorithm 3, there is a common expression " $\left(\partial V_{i} / \partial x\right)^{T} g(x)$ ", which is the only one in Algorithm 3 that explicitly contains the input coupling term $g(x)$. Considering the function $L_{\mu_{i}}(x)$ defined by the associated common term $\left(\partial V_{\mu_{i}} / \partial x\right)^{T} g(x)$ as

$$
L_{\mu_{i}}(x):=\left(\partial V_{\mu_{i}} / \partial x\right)^{T} g(x),
$$

one can obtain the following I-RL algorithm for the system (11) with the completely unknown dynamics:

\section{Algorithm 4: Model-free I-RL with Explorations}

\section{Policy Evaluation:}

$$
\begin{aligned}
V_{i}\left(x_{t}\right)+ & \int_{t}^{t+T} L_{i}(x) e_{\tau} d \tau \\
& =\int_{t}^{t+T} r\left(x(\tau), u_{i}(\tau)\right) d \tau+V_{i}\left(x_{t+T}\right)
\end{aligned}
$$

\section{Policy Improvement:}

$$
u_{i}(t)=-\frac{1}{2} R^{-1} L_{i-1}^{T}(x)
$$

Clearly, the equations (16) and (17) in Algorithm 4 do not contain any terms of $f(x)$ and $g(x)$ explicitly, which means that the iteration can be done without knowing the system dynamics $f(x)$ and $g(x)$.

Example 1: In case of linear quadratic regulation (LQR), the HJB equation (5) becomes the well-known algebraic Riccati equation (ARE) with the linear optimal control $u^{*}=$ $-(1 / 2) R^{-1}\left(L^{*}\right)^{T} x$ and the quadratic optimal value function 
$V^{*}(x)=x^{T} P^{*} x$, where $\left(P^{*}\right)^{T}=P^{*}>0$ is the solution of the ARE and $L^{*}:=2 P^{*} B$. Therefore, the exact parameterizations $L_{i}(x)=x^{T} L_{i}$ and $V_{i}(x)=x^{T} P_{i} x$, with abuse of notations, give the following specific forms of (16) and (17):

\section{Policy Evaluation:}

$$
\begin{aligned}
x_{t}^{T} P_{i} x_{t} & +\int_{t}^{t+T} x_{\tau}^{T} L_{i} e_{\tau} d \tau \\
& =\int_{t}^{t+T} r\left(x(\tau), u_{i}(\tau)\right) d \tau+x_{t+T}^{T} P_{i} x_{t+T}
\end{aligned}
$$

Policy Improvement: $\quad u_{i}(t)=-\frac{1}{2} R^{-1} L_{i-1}^{T} x$

which is a modified version of integral Q-learning for LQR [19]. For this algorithm, the matrices $P_{i}$ and $L_{i}$ exactly converge to $P^{*}$ and $L^{*}$, respectively, under the uniqueness of the matrices $P_{i}$ and $L_{i}$ for all $i \in \mathbb{N} \cup\{0\}$.

\section{IMPLEMENTATIONS}

In this section, an implementation method of the proposed I-RL algorithms is discussed, which is based on the batch least squares and exact parameterizations. Assume that for each $i$ th iteration, $V_{i}(x)$ and $L_{i}(x)$ are represented in the following linear regression forms:

$$
\begin{aligned}
& V_{i}(x)=\left(w_{c}^{[i]}\right)^{T} \phi_{c}(x), \\
& L_{i}(x)=\left(w_{a}^{[i]}\right)^{T} \phi_{a}(x),
\end{aligned}
$$

where $\left(w_{c}^{[i]}\right) \in \mathbb{R}^{N_{c}}$ and $\left(w_{a}^{[i]}\right) \in \mathbb{R}^{N_{a}}$ are the unknown weight parameters which will be determined by the proposed I-RL methods; $\phi_{c}(x) \in \mathbb{R}^{N_{c}}$ and $\phi_{a}(x) \in \mathbb{R}^{N_{a}}$ are the continuously differentiable nonlinear functions known $a$ priori. Here, the subscripts ' $c$ ' and ' $a$ ' denotes the critic and actor, respectively. For the discussions, the following notion of the persistency of excitation in DT fashion is needed:

Definition 2: A bounded DT signal $s_{k} \in \mathbb{R}^{r}(k \in \mathbb{N} \cup\{0\})$ is persistently exciting of order $L \in \mathbb{N}$ if there exist $\beta_{1}, \beta_{2}>$ 0 such that for all $k \in \mathbb{Z}_{+}$,

$$
\beta_{1} I \leq \sum_{l=0}^{L-1} s_{k+l} s_{k+l}^{T} \leq \beta_{2} I .
$$

This notion of PE condition implies that if $s_{k}$ is persistently exciting of order $L$, then, the matrix $S_{k}(\bar{L})$ defined by

$$
S_{k}=\left[\begin{array}{llll}
s_{k} & s_{k+1} & \cdots & s_{k+\bar{L}-1}
\end{array}\right]
$$

is of full rank for all $\bar{L} \geq L$ and all $k \in \mathbb{Z}_{+}$, the notion of PE with order $L$ presented in [21]. Note that all the policy evaluations in Algorithms 2-4 are based on the 1-dimensional equations, but we should determine $N_{c}$-number of parameters $w_{c}^{[i]}$ for Algorithms 2-3 and $\left(N_{c}+N_{a}\right)$-number of parameters $\left(w_{c}^{[i]}, w_{a}^{[i]}\right)$ for Algorithm 4 for each policy evaluation step. This means that for the update of the parameters, one should use multiple number of data vectors to overcome this rankdeficiency problem. For Algorithm 2, an example of this datadriven method was presented in [13] and [15] based on the least squares.

\section{A. Implementation of Algorithm 3}

Now, the least squares data-driven implementation is extended to Algorithm 3 with the system (11) explored by $e_{t}$. The key equation of the least-squares implementation lies in the equation (14) of policy evaluation. If one substitute (18) and (19) into (14) and rearrange the equation, the following equivalent formula can be obtained:

$$
\left[\left.x_{k,[t, T]}\right|_{k=0}\right]^{T} w_{c}^{[i]}=\left.y_{k,[t, T]}\right|_{k=0},
$$

where the DT signal $x_{k,[t, T]}$ and $y_{k,[t, T]}$ are defined as

$$
\begin{aligned}
x_{k,[t, T]}:= & \phi_{c}\left(x_{t+k T}\right)-\phi_{c}\left(x_{t+(k+1) T}\right) \\
& +\int_{t+k T}^{t+(k+1) T}\left(\frac{\partial \phi_{c}^{T}(x)}{\partial x}\right) g\left(x_{\tau}\right) e_{\tau} d \tau, \\
y_{k,[t, T]}:= & \int_{t+k T}^{t+(k+1) T} r\left(x(\tau), u_{i}(\tau)\right) d \tau .
\end{aligned}
$$

Here, by employing the differential equation $\dot{V}_{t}=r\left(x_{t}, u_{t}\right)$ and $\dot{W}_{t}=\left(\partial \phi_{c}(x) / \partial x\right)^{T} g\left(x_{t}\right) e_{t}$, the data $x_{k,[t, T]}$ and $y_{k,[t, T]}$ can be rewritten as

$$
\begin{aligned}
& x_{k,[t, T]}=\phi_{c}\left(x_{t+k T}\right)-\phi_{c}\left(x_{t+(k+1) T}\right)+W_{t+(k+1) T}-W_{t+k T} \\
& y_{k,[t, T]}=V_{t+(k+1) T}-V_{t+k T} .
\end{aligned}
$$

Now, let $X_{[t, T]}(L)$ and $Y_{[t, T]}(L)$ be the collections of the data vectors with length $L$, defined as

$$
\begin{aligned}
X_{[t, T]}(L) & :=\left[\begin{array}{llll}
x_{0,[t, T]} & x_{1,[t, T]} & \cdots & x_{L-1,[t, T]}
\end{array}\right] \\
Y_{[t, T]}(L) & :=\left[\begin{array}{llll}
y_{0,[t, T]} & y_{1,[t, T]} & \cdots & y_{L-1,[t, T]}
\end{array}\right]
\end{aligned}
$$

Then, assuming $\bar{L}$-number of data is collected at $i$-th policy evaluation, where $\bar{L}$ is any natural number satisfying $\bar{L} \geq N_{c}$, then, by recursively applying (21) for $k=0,1,2, \cdots, L$ and combining them, one has the following matrix-vector formula:

$$
\left[X_{[t, T]}(\bar{L})\right]^{T} w_{c}^{[i]}=Y_{[t, T]}(\bar{L}) .
$$

Note that if the signal $x_{k,[t, T]}$ is PE with order $N_{c}$, then, by the above arguments, $X_{[t, T]}(\bar{L})$ is of full rank, that is, $\operatorname{rank}\left(X_{[t, T]}(\bar{L})\right)=N_{c}$ and thus, $w_{c}^{[i]}$ can be uniquely determined by the solution of the least squares:

$$
w_{c}^{[i]}=\left[X_{[t, T]}(\bar{L}) X_{[t, T]}^{T}(\bar{L})\right]^{-1} X_{[t, T]}(\bar{L}) Y_{[t, T]}(\bar{L}) .
$$




\section{B. Implementation of Algorithm 4}

Similarly to Algorithm 3, the same least squares data-driven approach can be applied to Algorithm 4 for the implementation. Note that substituting (18) and (19) into (16) yields the following rearranged form of (16):

$$
\left[\left.\Delta \phi_{k,[t, T]}^{c}\right|_{k=0}\right]^{T} w_{c}^{[i]}+\left[\left.\rho_{k,[t, T]}^{a}\right|_{k=0}\right]^{T} w_{a}^{[i]},=\left.y_{k,[t, T]}\right|_{k=0},
$$

where the DT signal $\Delta \phi_{k,[t, T]}^{c}$ and $\rho_{k,[t, T]}^{a}$ are defined as

$$
\begin{aligned}
& \Delta \phi_{k,[t, T]}^{c}:=\phi_{c}\left(x_{t+k T}\right)-\phi_{c}\left(x_{t+(k+1) T}\right), \\
& \rho_{k,[t, T]}^{a}:=\int_{t+k T}^{t+(k+1) T} \phi_{a}(x) e_{\tau} d \tau .
\end{aligned}
$$

Moreover, by defining $z_{k,[t, T]}$ and $\bar{w}^{[i]}$ as

$$
z_{k,[t, T]}:=\left[\begin{array}{c}
\Delta \phi_{k,[t, T]}^{c} \\
-\overline{-}-- \\
\rho_{k,[t, T]}^{a}
\end{array}\right], \quad \bar{w}^{[i]}:=\left[\begin{array}{c}
w_{c}^{[i]} \\
-\overline{w_{a}^{[i]}}
\end{array}\right],
$$

(24) can be compactly rewritten as

$$
\left[\left.z_{k,[t, T]}\right|_{k=0}\right]^{T} \bar{w}^{[i]}=\left.y_{k,[t, T]}\right|_{k=0} .
$$

Then, repeating the same procedure to Algorithm 3, one can see that if the signal $z_{k,[t, T]}$ is PE with order $N_{c}+N_{a}$, then, $\bar{w}^{[i]}$ can be uniquely evaluated by the least squares:

$$
\bar{w}^{[i]}=\left[Z_{[t, T]}(\bar{L}) Z_{[t, T]}^{T}(\bar{L})\right]^{-1} Z_{[t, T]}(\bar{L}) Y_{[t, T]}(\bar{L}),
$$

where $\bar{L}$ is any number satisfying in this case $\bar{L} \geq N_{c}+N_{a}$; $Z_{[t, T]}(L)$ is the collection of the data vectors defined as

$$
Z_{[t, T]}(L):=\left[\begin{array}{llll}
z_{0,[t, T]} & z_{1,[t, T]} & \cdots & z_{L-1,[t, T]}
\end{array}\right] .
$$

Remark 2: The previous discussions show that the solvability of the least squares (23) and (26) depends on the order of the PE condition of $x_{k,[t, T]}$ and $z_{k,[t, T]}$-if $x_{k,[t, T]}$ and $z_{k,[t, T]}$ are PE with order $N_{c}$ and $N_{c}+N_{a}$, then, the unique solution $w_{c}^{[i]}$ and $\bar{w}^{[i]}$ can be obtained by (23) and (26), respectively.

\section{Numerical Simulations}

To verify the effectiveness of the proposed algorithms, we have conducted numerical simulations for the 2-dimensional nonlinear system given by

$$
\begin{aligned}
& f(x)=\frac{1}{2}\left[\begin{array}{c}
-(3 / 2) x_{1}+x_{2} \\
\left(2 x_{1}+3 x_{2}\right) \sin ^{2} x_{1}+x_{1}-x_{2}
\end{array}\right] \\
& g(x)=\left[\begin{array}{ll}
0 & \sin x_{1}
\end{array}\right]^{T}
\end{aligned}
$$

with the performance index $r(x, u)=x^{T} x+u^{2}$. By converse HBJ approach [22], the optimal value function $V^{*}(x)$ and policy $u^{*}=\mu^{*}(x)$ is given by

$$
\begin{aligned}
& V^{*}(x)=2 x_{1}^{2}+4 x_{1} x_{2}+3 x_{2}^{2}, \\
& \mu^{*}(x)=-2 x_{1} \sin x_{1}-3 x_{2} \sin x_{1} .
\end{aligned}
$$



Fig. 1. The evolutions of the critic weight $w_{c}^{[i]}$ for explorized I-RL

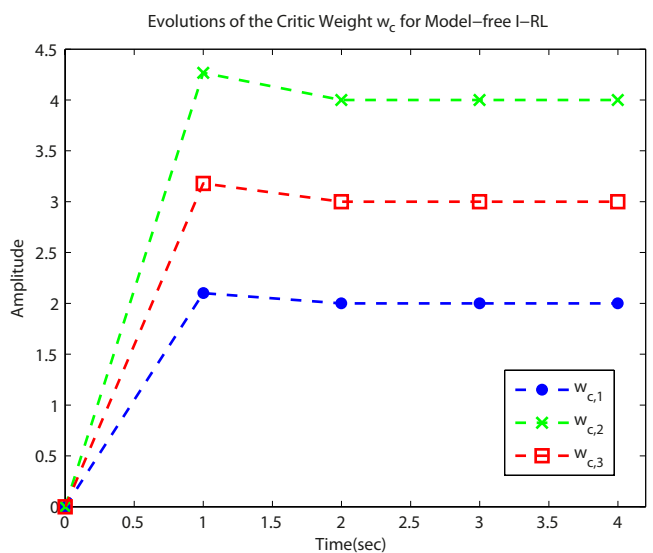

Fig. 2. The evolutions of the critic weight $w_{c}^{[i]}$ for model-free I-RL

So, the basis function of $V_{i}(x)$ and $L_{i}(x)$ can be chosen as

$$
\begin{aligned}
\phi_{c}(x) & =\left[\begin{array}{lll}
x_{1}^{2} & x_{1} x_{2} & x_{2}^{2}
\end{array}\right]^{T}, \\
\phi_{a}(x) & =\left[\begin{array}{ll}
x_{1} \sin x_{1} & x_{2} \sin x_{1}
\end{array}\right]^{T},
\end{aligned}
$$

respectively. This exactly parameterize $V^{*}(x)$ and $L^{*}(x)$ as $V^{*}(x)=\left(w_{c}^{*}\right)^{T} \phi_{c}(x)$ and $L^{*}(x)=\left(w_{a}^{*}\right)^{T} \phi_{a}(x)$ with the optimal weights $w_{c}^{*}=\left[\begin{array}{lll}2 & 4 & 3\end{array}\right]^{T}$ and $w_{a}^{*}=\left[\begin{array}{ll}4 & 6\end{array}\right]^{T}$. From these parametric structures, $N_{c}$ and $N_{a}$ are determined as $N_{c}=3$ and $N_{a}=5$, so that we conducted the simulations with $\bar{L}=5$ for Algorithm 3 and $\bar{L}=10$ for Algorithm 4 . For both simulations, we set the initial condition and weights as $x_{0}=\left[\begin{array}{ll}0.1 & -0.1\end{array}\right]^{T}, w_{c}^{[0]}=0$, and $w_{a}^{[0]}=0$; the period $T=0.1$ [s] and exploration $e_{t}=5 \sin (2 \pi t)$ are used in the learning process. Note that with these settings, the parameters are updated every $T \bar{L}=0.5[\mathrm{~s}]$ for Algorithm 3 and every $T \bar{L}=1$ [s] for Algorithm 4 .

Figs. 1-2 illustrate the evolutions of the critic weights $w_{c}^{[i]}$ for explorized I-RL and model-free I-RL, respectively. As can be seen, the weights converge to the optimal values after several iterations. Here, note that both represent the same results in iteration domain. In time domain, the only difference is the update period. Fig. 3 shows the variations of the actor weight $w_{a}^{[i]}$ for the model-free I-RL, which also 


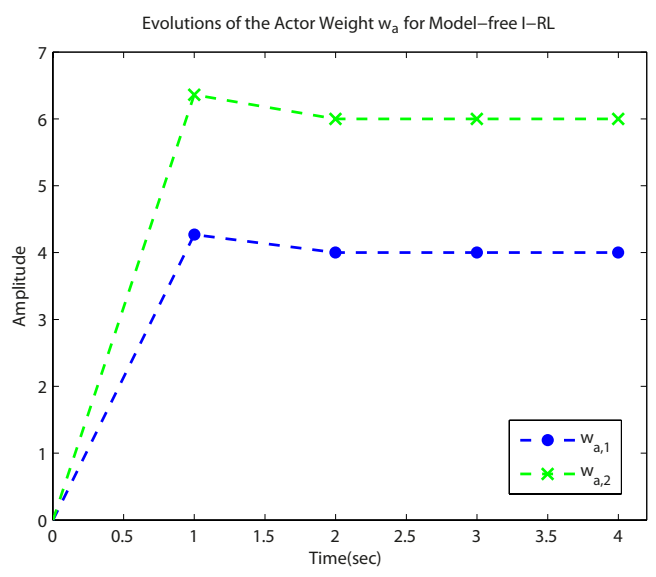

Fig. 3. The evolutions of the actor weight $w_{a}^{[i]}$ for model-free I-RL

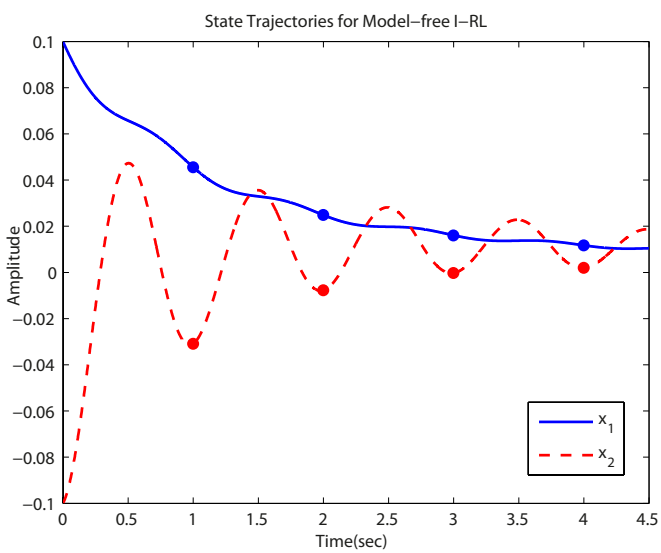

Fig. 4. The state trajectories for model-free I-RL

converge to the optimal one after several steps, implying the convergence of $L_{i}(x)$ to $L^{*}(x)$. During the learning procedure, the states remain stable but are rather ocillatory due to both the exploration $e_{t}$ and the transient policy that is initially not optimal but converges to the optimal one.

\section{CONCLUding REMARKS}

In this paper, two I-RL methods named respectively as "explorized I-RL" and "model-free I-RL" were proposed for CT input-affine nonlinear systems. Both methods were derived from the policy-iteration-based I-RL and effectively used the explorations in the policy evaluation step. Furthermore, in case of model-free I-RL, the use of exploration relaxed the need for the knowledge of input coupling dynamics, making the algorithm model-free. The implementation methods of these algorithms were presented with the connection to the PE conditions. Here, the exact parameterizatoin were assumed for the implementation, which is not satisfied in general. Therefore, the future works would be to develop the method with function approximators such as neural networks that can uniformly approximate any continuously differentiable function to a desired level [14], [15], and then, provide its stability and convergence.

\section{ACKNOWLEDGMENT}

This work was supported by the Brain Korea 21 Project in 2011 and by the Human Resources Development of the Korea Institute of Energy Technology Evaluation and Planning (KETEP) grant funded by the Korea government Ministry of Knowledge Economy. (No. 20104010100590).

\section{REFERENCES}

[1] R. S. Sutton and A. G. Barto, Reinforcement Learning-An Introduction, MIT Press, Cambridge, Massachussetts, 1998.

[2] J. Si, A. G. Barto, W. B. Powell, and D. Wunsch, Handbook of Learning and Approximate Dynamic Programming, Wiley-IEEE Press, 2004.

[3] F. Y. Wang, H. Zhang, and D. Liu, "Adaptive dynamic programming: an introduction," IEEE Computational Magazine, vol. 4, no. 2, pp. 39-47, 2009.

[4] F. L. Lewis and D. Vrabie, "Reinforcement learning and adaptive dynamic programming for feedback control," IEEE Circuits and Systems Magazine, vol. 9, no. 3, pp. 32-50, 2009.

[5] D. V. Prokhorov and D. C. Wunsch II, "Adaptive critic designs," IEEE Trans. Neural Networks, vol. 8, no. 5, pp. 997-1007, 1997.

[6] S. J. Bradtke and B. E. Ydstie, "Adaptive linear quadratic control using policy iteration," Proc. American Control Conference, Baltimore, Maryland, pp. 3475-3479, 1994.

[7] A. Al-Tamimi, M. Abu-Khalaf, and F. L. Lewis, "Adaptive critic designs for discrete-time zero-sum games with application to $H_{\infty}$ control," IEEE Trans. Syst., Man, Cybern.-Part B, vol. 37, no. 1, pp. 240-247, 2007.

[8] A. Al-Tamimi, M. Abu-Khalaf, and F. L. Lewis, "Model-free $Q$-learning designs for discrete-time zero-sum games with application to $H_{\infty}$ control," Automatica, vol. 43, no. 3, 473-481, 2007.

[9] A. Al-Tamimi, F. L. Lewis, and M. Abu-Khalaf, "Discrete-time nonlinear HJB solution using approximate dynamic programming: convergence proof," IEEE Trans. Systems, Man, and Cybernetics-PART B: Cybernetics, vol. 38, no. 4, 2008.

[10] K. Doya, "Reinforcement learning in continuous-time and space," Neural Computation 12, pp. 219-245, 2000.

[11] T. Hanselmann, L. Noakes, and A. Zaknich, "Continuous-time adaptive critics," IEEE Trans. Neural Network, vol. 18, no. 3, pp. 631-647, 2007.

[12] J. J. Murray, C. J. Cox, G. G. Lendaris, and R. Saeks, "Adaptive Dynamic Programming," IEEE Trans. Systems, Mans and CyberneticsPART B: Cybernetics, vol. 32, no. 2, pp. 140-153, 2002.

[13] D. Vrabie, O. Pastravanu, M. Abu-Khalaf, and F. L. Lewis, "Adaptive optimal control for continuous-time linear systems based on policy iteration," Automatica, vol. 45, no. 2, pp. 477-484, 2009.

[14] M. Abu-Khalaf and F. L. Lewis, "Nearly optimal control laws for nonlinear systems with saturating actuators using a neural network HJB approach," Automatica, vol. 41, no. 5, pp. 779-791, 2005.

[15] D. Vrable and F. L. Lewis "Neural network approach to continuous-time direct adaptive optimal control for partially unknown nonlinear systems," Neural Networks, vol. 22, no. 3, pp. 237-246, 2009.

[16] K. G. Vamvoudakis, F. L. Lewis "Online actor-critic algorithm to solve the continuous-time infinite horizon optimal control problem," Automatica, pp. 878-888 vol. 46, no. 5, pp. 878-888, 2010.

[17] S. Bhasin, M. Johnson, W. E. Dixon, "A model-free robust policy iteration algorithm for optimal control of nonlinear systems," 49th IEEE Conf. Decision and Control, Atlanta, GA, pp. 3060-3065, 2010.

[18] J. Y. Lee, J. B. Park, and Y. H. Choi, "Policy-iteration-based adaptive optimal control for uncertain continuous-time linear systems with excitation signals," in Proc. Int'l Conf. on Control, Automation, and Systems (ICCAS), Ilsan, Kyonggi-Do, South Korea, pp. 646-651, Oct. 2010.

[19] J. Y. Lee, J. B. Park, and Y. H. Choi, "Integral Q-learning and explorized policy iteration for adaptive optimal control of continuous-time linear systems," Automatica, accepted for publication, 2012.

[20] G. N. Saridis and C. G. Lee, "An approximation theory of optimal control for trainable manipulators," IEEE Trans. Systems, Man, and Cybernetics-PART B: Cybernetics, vol. 9, no. 3, 1979.

[21] J. C. Willems, P. Rapisarda, I. Markovsky, and Bart L. M. Moor, "A note on persistency of excitation," Systems \& Control Letters, vol. 54, no. 4, pp. 325-329, 2005.

[22] V. Nevistic and J. A. Primbs, "Constrained nonlinear optimal control: a converse HJB approach,” Technical report CIT-CDS 96-021, California Institute of Technology, Pasadena, CA 91125, 1996 